Kansas State University Libraries

New Prairie Press

\title{
ANALYSIS OF THE SPATIAL DISTRIBUTION OF SUGARBEET PLANTS
}

Stephen D. Kachman

John A. Smith

Follow this and additional works at: https://newprairiepress.org/agstatconference

Part of the Agriculture Commons, and the Applied Statistics Commons

\section{(c) (1) $\Theta($}

This work is licensed under a Creative Commons Attribution-Noncommercial-No Derivative Works 4.0 License.

\section{Recommended Citation}

Kachman, Stephen D. and Smith, John A. (1991). "ANALYSIS OF THE SPATIAL DISTRIBUTION OF SUGARBEET PLANTS," Conference on Applied Statistics in Agriculture. https://doi.org/10.4148/ 2475-7772.1423

This is brought to you for free and open access by the Conferences at New Prairie Press. It has been accepted for inclusion in Conference on Applied Statistics in Agriculture by an authorized administrator of New Prairie Press. For more information, please contact cads@k-state.edu. 


\title{
ANALYSIS OF THE SPATIAL DISTRIBUTION OF SUGARBEET PLANTS
}

\author{
Stephen D. Kachman \\ Department of Biometry \\ University of Nebraska-Lincoln
}

\author{
John A. Smith \\ Panhandle Research \& Extension Center \\ University of Nebraska-Lincoln
}

\begin{abstract}
The spatial distribution of emerged sugarbeet plants is an important aspect of the performance of sugarbeet planters. Three major components influencing the spatial distribution are the ability to drop a single seed at a time, the ability to drop the seeds a fixed distance apart, and the ability of the seed to emerge. A model has been developed to describe the distribution of the spacing between emerged sugarbeet plants. The model consists of a mixture of normal and gamma distributions. The spatial data consists of the distance between neighboring emerged plants. Spatial data was collected on 7 planters operated at 3 speeds using both pelleted and encrusted seeds. Four replicates were obtained of each treatment combination. Approximate maximum likelihood estimates of the parameters were obtained separately for each replicate of the treatment combinations.
\end{abstract}

Key Words: Gamma, Maximum likelihood, Sugarbeet, Planter.

\section{Introduction}

To compare planters with regard to spacing performance a model describing the spatial distribution is needed. One method of modeling planter performance is through the use of a histogram. A histogram is very general with the probability of seedlings falling within a given range of distances apart being estimated separately for each range. The histogram is very useful in graphically summarizing planting performance.

However, one is usually interested in quantifying various aspects of the shape of the spatial distribution. A more restrictive model focuses on certain features of the shape of the distribution while ignoring or down playing others. A model should therefore capture the features of the spatial distribution which are important in judging planter performance. It is not necessary however, that the model be the true model underlying planter spacing, only that it do a reasonable job approximating the true model. A model that is too general will probably suffer from problems associated with estimating too many parameters given the data.

A number of alternative approaches have been used to describe the spatial distribution in sugarbeets. Rohrbach, Brazee and Barre (1969) modeled the spatial distribution of uniform plant spacing devices as a mixture of normal densities. Rohrbach, Brazee and Barre (1971) examined the performance of the model using field data for a variety of planters and crops. For all the crops examined, except sugarbeets, the model appeared to perform well. However, in three of the four field tests involving sugarbeets there were significant deviations between the actual and predicted frequencies $(\mathrm{P}<.01)$.

The objective of this paper is to develop a method of quantifying a planter's ability to accurately place sugarbeet seeds. In Section 2 a model will be developed to describe the spacing of sugarbeet plants in a row. The parameters from the model describe various aspects of planter performance. In Section 3 an estimation procedure will be developed to estimate the unknown parameters. In Section 4 spacing data from an experiment conducted at the Panhandle Research and Extension Center in Scottsbluff, Nebraska will be used to demonstrate how the estimated parameters can be used to quantify a planter's ability to accurately place sugarbeet seeds. 


\section{Model}

A number of models are possible to describe the spacing performance of planters. The model that will be developed here will based on three factors affecting planter performance. The first factor is the ability of the planter to select a single seed. A planter that has a greater tendency to select multiple seeds will produce a cluster of spacings near zero. However, a planter which has a greater tendency to fail to select a seed will have a greater tendency to produce clusters of spacings around two or more times the desired distance. The second factor is the ability of the planter to release the selected seeds at the predetermined distance. A planter which does a better job of the releasing seeds at the predetermined distance should produce a tight cluster of spacings around the predetermined distance and at two or more times the predetermined distance. The third factor is the ability of the planted seed to develop into a seedling. Seeds which have a greater tendency of developing into a seedling will have a greater tendency to produce cluster of spacings around the desired distance.

The model will be developed in two stages. In the first stage, a model for the seed selection mechanism will be presented. In the second stage, a model for the distance between the seeds will be presented. Because the data does not differentiate between a seed that does not drop and a seed the does not emerge, the model pertaining to seed emergence is merged with the model for number of seeds selected.

\subsection{Seed selection}

The first factor to be modeled is the ability of the planter to select and drop a single seed. The probability that a planter will fail to drop at least one seed will be given by $p$. It is important to note that for our purposes a seed that does not emerge will be treated the same as a seed that was never dropped. Let $a$ be the number of times the planter attempted to drop a seed since the last seed was dropped. In other words, if a seed was dropped on the first attempt then the number of attempts would be one.

Major factors contributing to a seed failing to drop are a failure of the seed selection mechanism, the failure of a seed to be released, and the failure of the seed to emerge. These factors will tend to be influenced by factors particular to that attempt. We will assume that a successful attempt at dropping a seed is independent of whether previous attempts have been successful. The probability of $a$ attempts between drops of one or more seeds is therefore given by the following geometric probability function

$$
P(A=a \mid A>0)=(1-p) p^{a-1} .
$$

The average number of attempts between drops is $1 /(1-p)$.

Occasionally more than a single seed is dropped at a time. Even rarer is for more than two seeds to be dropped at the same time. A discrete distribution for the number of seeds dropped is needed. It should, given that the probability of multiple seeds dropping is small, have the probability of more than two seeds dropping be very small. The geometric probability function has this property. Assuming that at least one seed is dropped the probability of $n$ seeds dropping is given by the following geometric probability function

$$
P(N=n \mid N>0)=\left(1-p_{0}\right) p_{0}^{n-1}
$$

where $p_{0}$ is the probability that when a seed is dropped that more than one seed is dropped. It will also be assumed that the number of seeds that are dropped at a given point is independent of the number of seeds dropped at other locations.

Combining the distributions for multiple seeds drops and attempts between drops we end up with the following probability function for the number of seeds dropped

$$
P(N=n)=\left\{\begin{array}{cc}
p & n=0 \\
(1-p)\left(1-p_{0}\right) p_{0}^{n-1} & n>0
\end{array} .\right.
$$


The average number of seeds dropped, including 0 seeds dropped, is $(1-p) /\left(1-p_{0}\right)$.

The number of attempts between two seeds is

$$
P(A=a)=\left\{\begin{array}{cc}
1-p_{0} & a=0 \\
p_{0}(1-p) p^{a-1} & a>0
\end{array}\right.
$$

where $a=0$ attempts implies the two seeds were dropped at the same time.

\subsection{Distance between attempted seed drops}

The second factor to be modeled is the ability of the planter to drop the selected seeds at the predetermined distance. The distance between attempted seed drops will be measured as the distance between the end of the last seed drop and the beginning of the next seed drop.

A number of factors affect the distance between seed drops. In sugarbeets these factors combine to introduce a considerable amount of variability. We would therefore expect the distribution of distances between seed spacings to be skewed right. Given the relative rare occurrence of multiples, we will assume that the distance between seed drops is not a function of the number of seeds dropped. A gamma distribution was selected to model the distribution of the distance between attempted seed drops $d$. The density is

$$
f(d)=\frac{d^{\alpha-1} \exp (-d / \beta)}{\Gamma(\alpha) \beta^{\alpha}}
$$

where $\alpha$ is a shape parameter and $\beta$ is a scale parameter. The average distance between attempted drops is given by $\alpha \beta$ and the variance is given by $\alpha \beta^{2}$. The coefficient of variation for the distance between attempted drops is given by $1 / \sqrt{\alpha}$.

The absolute value of normal random variable centered at zero will be used to model the distance between seeds dropped at the same time. The density is given as

$$
f_{0}(d)=\sqrt{\frac{2}{\pi \sigma_{0}^{2}}} \exp \left(\frac{-d^{2}}{2 \sigma_{0}^{2}}\right)
$$

where $\sigma_{0}^{2}$ is the variance of the original normal random variable. The absolute value of a normal random variable was selected because if two seeds are dropped at the same time and their distances down the row are distributed as independent normal random variables with mean $\mu$ and variance $\sigma_{0}^{2} / 2$, then the distance between the two seeds will be distributed as $f_{0}(d)$. Given that multiples in excess of doubles appear to be very infrequent no attempt was taken to model it.

No attempt was taken to adjust the distribution of the distance between attempted seed drops for the number of seeds dropped. The number of seeds dropped factor was ignored, because the variability around the attempted seed drop point is usually small compared to the distance between the seed drop points. In addition, when the variability around a seed drop point is not small compared to the distance between attempted drop points the noise in the data would be expected to overwhelm any possible gains obtained by fine tuning the model.

\section{Estimation}

Approximate maximum likelihood estimates of the unknown parameters $\left(p, p_{0}, \sigma_{0}^{2}, \alpha, \beta\right)$ were obtained using the EM algorithm (Dempster, Laird and Rubin, 1977) and an approximation of the likelihood function. The EM algorithm consists of iterating between two steps. In the first step, the expected value of the log likelihood function for the complete data conditional on the incomplete data and the previous estimates of the parameters is obtained. In the second step, the parameters which would maximize the expected value of the log likelihood are found. 
The incomplete data consisted of the spacing between the plants $(z)$. The complete data consisted of the spacing between the plants along with the number of attempted drops since the last seed was dropped. The log likelihood function for the complete data is

$$
\begin{aligned}
& L(\theta \mid x)=\sum_{i=1}^{N} {\left[(1 / 2)\left\{2 \ln \left(1-p_{0}\right)+\ln (2 / \pi)-\ln \left(\sigma_{0}^{2}\right)+\left(\frac{-z_{i}^{2}}{\sigma_{0}^{2}}\right)\right\} I_{a_{i}=0}\right.} \\
&+\left\{\ln \left(p_{0}\right)+\ln (1-p)+\left(a_{i}-1\right) \ln (p)\right. \\
&\left.\left.+\left(a_{i} \alpha-1\right) \ln \left(z_{i}\right)-z_{i} / \beta-\ln \left(\Gamma\left(a_{i} \alpha\right)\right)-a_{i} \alpha \ln (\beta)\right\} I_{a_{i}>0}\right]
\end{aligned}
$$

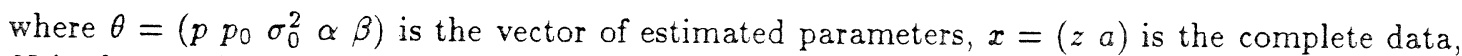
$N$ is the number of spacings, $a_{i}=0$ if the two seeds were dropped at the same time, $a_{i}>0$ if the two seeds were not dropped at the same time, and $I$ is the indicator function.

\subsection{Expectation step}

The expected value of log likelihood function is

$$
\begin{aligned}
Q(\theta \mid z)=\sum_{i=1}^{N} & {\left[(1 / 2)\left\{2 \ln \left(1-p_{0}\right)+\ln (2 / \pi)-\ln \left(\sigma_{0}^{2}\right)+\left(\frac{-z_{i}^{2}}{\sigma_{0}^{2}}\right)\right\} \operatorname{Pr}\left(a_{i}=0 \mid z_{i}\right)\right.} \\
+ & \sum_{j=1}^{\infty}\left\{\ln \left(p_{0}\right)+\ln (1-p)+(j-1) \ln (p)\right. \\
& \left.+(j \alpha-1) \ln \left(z_{i}\right)-z_{i} / \beta-\ln (\Gamma(j \alpha))-j \alpha \ln (\beta)\right\} \\
& \left.\cdot \operatorname{Pr}\left(a_{i}=j \mid z_{i}\right)\right],
\end{aligned}
$$

where

$$
\operatorname{Pr}\left(a_{i}=j \mid z_{i}\right) \propto\left\{\begin{array}{ll}
\left(1-p_{0}\right) \sqrt{\frac{2}{\pi \sigma_{0}^{2}}} \exp \left(\frac{-z_{i}^{2}}{2 \sigma_{0}^{2}}\right) & j=0 \\
p_{0}(1-p) p^{j-1} \frac{z_{i}^{j \alpha-1} \exp \left(-z_{i} / \beta\right)}{\Gamma(j \alpha) \beta^{j \alpha}} & j>0
\end{array} .\right.
$$

The $\operatorname{Pr}\left(a_{i}=j \mid z_{i}\right)$ is evaluated using estimates of the unknown parameters. The log gamma function will be approximated using Stirling's approximation (Abramowitz and Stegun, 1972, eq. 6.1 .41 ) given below

$$
\ln (\Gamma(x)) \approx(x-1 / 2) \ln (x)-x+1 / 2 \ln (2 \pi)
$$

As seen in Figure 1 the approximation is very good except for values close to zero. In the cases where $\alpha$ is close to zero the coefficient of variation $(1 / \sqrt{\alpha})$ is large and would be indicative of a very poor planter performance. Using this approximation for the log gamma function will allow the maximization of the approximate $Q(\theta \mid z)$ to be obtained directly.

The approximate $Q(\theta \mid z)$ is

$$
\begin{aligned}
Q(\theta \mid z)=\sum_{i=1}^{N} & {\left[(1 / 2)\left\{2 \ln \left(1-p_{0}\right)+\ln (2 / \pi)-\ln \left(\sigma_{0}^{2}\right)+\left(\frac{-z_{i}^{2}}{\sigma_{0}^{2}}\right)\right\} \operatorname{Pr}\left(a_{i}=0 \mid z_{i}\right)\right.} \\
& +\sum_{j=1}^{\infty}\left\{\ln \left(p_{0}\right)+\ln (1-p)+(j-1) \ln (p)\right. \\
& +(j \alpha-1) \ln \left(z_{i}\right)-z_{i} / \beta-(j \alpha-1 / 2) \ln (j \alpha) \\
& \left.+j \alpha-1 / 2 \ln (2 \pi)-j \alpha \ln (\beta)\} \operatorname{Pr}\left(a_{i}=j \mid z_{i}\right)\right] .
\end{aligned}
$$


After some rearranging we get

$$
\begin{aligned}
Q(\theta \mid z)=\sum_{i=1}^{N} & {\left[(1 / 2)\left\{2 \ln \left(1-p_{0}\right)+\ln (2 / \pi)-\ln \left(\sigma_{0}^{2}\right)+\left(\frac{-z_{i}^{2}}{\sigma_{0}^{2}}\right)\right\} \operatorname{Pr}\left(a_{i}=0 \mid z_{i}\right)\right.} \\
& +\left\{\left(\ln \left(p_{0}\right)+\ln (1-p)-\ln (p)+1 / 2 \ln (\alpha)-\ln \left(z_{i}\right)-z_{i} / \beta-1 / 2 \ln (2 \pi)\right)\right. \\
& +\left(\ln (p)+\alpha+\alpha \ln \left(z_{i} / \beta\right)-\alpha \ln (\alpha)\right) \mathrm{E}\left(a_{i} \mid z_{i}, a_{i}>0\right) \\
& \left.\left.+(1 / 2) \mathrm{E}\left(\ln \left(a_{i}\right) \mid z_{i}, a_{i}>0\right)-\alpha \mathrm{E}\left(a_{i} \ln \left(a_{i}\right) \mid z_{i}, a_{i}>0\right)\right\} \operatorname{Pr}\left(a_{i}>0 \mid z_{i}\right)\right]
\end{aligned}
$$

\subsection{Maximization step}

The value of the parameters that maximizes $Q(\theta \mid z)$ will be obtained by setting the first partials of $Q(\theta \mid z)$ with respect to the parameters equal to zero. The first partials are given in Appendix $A$.

Setting the first partials equal to zero and solving, yield the following estimation equations

$$
\begin{aligned}
\hat{p}_{0} & =\frac{\sum_{i=1}^{N} \operatorname{Pr}\left(a_{i}>0 \mid z_{i}\right)}{N} \\
\hat{\sigma}_{0}^{2} & =\frac{\sum_{i=1}^{N} z_{i}^{2} \operatorname{Pr}\left(a_{i}=0 \mid z_{i}\right)}{N\left(1-\widehat{p}_{0}\right)} \\
\hat{p} & =1-\frac{\sum_{i=1}^{N} \operatorname{Pr}\left(a_{i}>0 \mid z_{i}\right)}{\sum_{i=1}^{N} E\left(a_{i} \mid z_{i}, a_{i}>0\right) \operatorname{Pr}\left(a_{i}>0 \mid z_{i}\right)} \\
\hat{\alpha} & =\frac{(1 / 2) N \hat{p}_{0}}{\ln \left(\frac{\sum_{i=1}^{N} z_{i} \operatorname{Pr}\left(a_{i}>0 \mid z_{i}\right)}{\sum_{i=1}^{N} \mathrm{E}\left(a_{i} \mid z_{i}, a_{i}>0\right) \operatorname{Pr}\left(a_{i}>0 \mid z_{i}\right)}\right)+\sum_{i=1}^{N} \mathrm{E}\left(a_{i} \ln \left(a_{i} / z_{i}\right) \mid z_{i}, a_{i}>0\right) \operatorname{Pr}\left(a_{i}>0 \mid z_{i}\right)} \\
\hat{\beta} & =\frac{\sum_{i=1}^{N} z_{i} \operatorname{Pr}\left(a_{i}>0 \mid z_{i}\right)}{\hat{\alpha} \sum_{i=1}^{N} \mathrm{E}\left(a_{i} \mid z_{i}, a_{i}>0\right) \operatorname{Pr}\left(a_{i}>0 \mid z_{i}\right)} .
\end{aligned}
$$

It should be noted that both the expected values and probabilities are evaluated using the previous estimates of the parameters.

\section{1990 Spacing Data}

In 1990 spacing data on seven sugarbeet planters were collected at the Panhandle Research and Extension Center in Scottsbluff Nebraska. The 1990 spacing data were used to determine if the estimates from the above model could be used to quantify differences between planters under various operating conditions. The seven planters that were compared were a Stanhay S870 Belt, a White Seedboss, a Stanhay Rallye 590, a Deere 71, a Gaspardo SV255, a Deere Maxemerge II, and a Milton. There were 6 rows for each of the planters except for the White Seedboss and Deere Maxemerge II which shared the same tool bar having 4 rows (rows one through four) and 2 rows (rows five and six) respectively and the Gaspardo SV255 which was a 4 row planter. Each planter was operated at 3 speeds and with 2 types of seeds. The speeds were 2, 3.5 and 5 miles per hour and the seed types were encrusted and pelleted. The Stanhay S870 Belt and the White Seedboss were each used with just a single seed type, pelleted and encrusted respectively.

Four replications of each of the planter, speed and seed type combinations were obtained in a split-plot design. Treatment combinations involving a single seed type were randomized within each plot. On each row of the planter two subsamples of 30 consecutive seeds were taken. Distances between the seeds were recorded to the nearest centimeter resulting in 29 distances for each of two subsamples. The two subsamples within a row were combined into a single sample. Estimates of the parameters $\left(p_{0}, \sigma_{0}^{2}, p, \alpha, \beta\right)$ were obtained for each of the samples. The 
estimated parameters along with the coefficient of variation $(C V=1 / \sqrt{\alpha})$ and mode $((\alpha-1) \beta)$ were then treated as the traits of interest.

The model was

$$
\begin{aligned}
& y_{i j k l m n}=\mu+\text { Type }_{i}+\operatorname{Rep}\left(\text { Type }_{i j}+\text { Planter }_{k}+\text { Type }_{\text {Planter }} \text { P } \text { P }_{k}+\text { Speed }_{l}+\right. \\
& \text { Type } * \text { Speed }_{i l}+\text { Planter } \text { Speed }_{k l}+\text { Type }^{*} \text { Planter } * \text { Speed }_{i k l}+\text { Side }_{m}+
\end{aligned}
$$

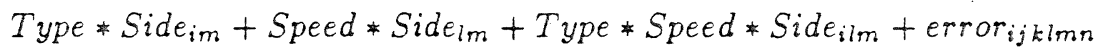

where $y_{i j k l m}$ is the average of estimated parameter for the rows on side $m$ of the tool bar, Type is the effect of seed type $i, \operatorname{Rep}(\text { Type })_{i j}$ is the effect of replicate $j$ of seed type $i$, Planter $_{k}$ is the effect of planter $k$, Speed $d_{l}$ is the effect of planting at speed $l$, Side $e_{m}$ is the effect side $m$ (rows one through four, or rows five and six) and error $r_{i j k i m n}$ is the residual. The Rep(Type $)_{i j}$ s were assumed to be be distributed as $N\left(0, \sigma_{R / T}^{2}\right)$. The error ${ }_{i j l k m n} \mathrm{~s}$ were assumed to be distributed as $N\left(0, \sigma_{\varepsilon}^{2} / n_{i j k l m n}\right)$, where $n_{i j k l m n}$ is the number of rows on side $m$ of the tool bar.

\section{Results of the 1990 Spacing Data Analysis}

As seen in Figures 2 and 3 the model was able to describe a wide range of spacing distributions. Two problem areas were found when the estimated parameters were averaged across samples. First, when the estimated probability of multiples $\left(p_{0}\right)$ was close to zero the estimated variance $\left(\sigma_{0}^{2}\right)$ tended to get very large. This relationship is to be expected given that a lack of very small spacings can be accounted for by either a very small probability of multiples or a very large variance. Second, when the estimated coefficient of variation $(C V)$ was large the estimated skip probability $(p)$ tended to be small. This relationship may be due to the inability of the model to separate the different gamma distributions when the are poorly defined as in Figure 3.

The results of the analysis for the coefficient of variation, mode, probability of multiples $\left(p_{0}\right)$, and the probability of a skip $(p)$ are summarized in Table I. Planter, speed of operation, and seed type each had significant effects on each of the measure of planter performance. With the exception of the mode, side of the tool bar did not have a significant effect on planter performance.

Least square means for encrusted and pelleted seeds are given in Tables II and III respectively. With very few exceptions it appears that as the speed at which the planter is operated increases the ability of the planter to accurately place seeds decreases. Interactions between planters and speed of operation appears to be in at which speed does planter performance deteriorate. With the exception of the Gaspardo SV255 the planters appear to have less trouble placing pelleted seeds.

\section{Summary}

A model consisting of a mixture of normal and gamma distributions was developed to describe the spatial distribution of sugarbeet plants. The model along with the estimation procedure appears to do a good job in modeling a wide range of spatial distributions that can arise. The estimated parameters from the model provide an objective measure of planter performance. However, it appears that in some situations the estimated density may be biased (e.g. the second peak in Figure 2). The bias may be a result of using samples of 58 spacings to estimate the parameters. In addition, it appears an estimate of the standard errors associated with the estimated parameters are needed.

\section{References}

Abramowitz, M. and Stegun, I. A., eds. (1972), Handbook of mathematical functions with formulas, graphs, and mathematical tables (ninth printing), Dover, Mineola, NY. 
estimated parameters along with the coefficient of variation $(C V=1 / \sqrt{\alpha})$ and mode $((\alpha-1) \beta)$ were then treated as the traits of interest.

The model was

$$
\begin{aligned}
& y_{i j k l m n}=\mu+\text { Type }_{i}+\operatorname{Rep}\left(\text { Type }_{i j}+\text { Planter }_{k}+\text { Type } \text { Planter }_{i k}+\text { Speed }_{l}+\right. \\
& \text { Type } * \text { Speed }_{i l}+\text { Planter } * \text { Speed }_{k l}+\text { Type* Planter } * \text { Speed }_{i k l}+\text { Side }_{m}+ \\
& \text { Type } * \text { Side }_{i m}+\text { Speed } * \text { Side }_{l m}+\text { Type } \text { Speed } * \text { Side } e_{i m}+\text { error }_{i j k l m n}
\end{aligned}
$$

where $y_{i j k l m}$ is the average of estimated parameter for the rows on side $m$ of the tool bar, Type; is the effect of seed type $i, R e p(T y p e)_{i j}$ is the effect of replicate $j$ of seed type $i$, Planter $_{k}$ is the effect of planter $k$, Speed $_{l}$ is the effect of planting at speed $l$, Side ${ }_{m}$ is the effect side $m$ (rows one through four, or rows five and six) and error $r_{i j k l m n}$ is the residual. The Rep(Type $)_{i j}$ s were assumed to be be distributed as $N\left(0, \sigma_{R / T}^{2}\right)$. The error $i_{i j l k m n}$ s were assumed to be distributed as $N\left(0, \sigma_{e}^{2} / n_{i j k i m n}\right)$, where $n_{i j k ! m n}$ is the number of rows on side $m$ of the tool bar.

\section{Results of the 1990 Spacing Data Analysis}

As seen in Figures 2 and 3 the model was able to describe a wide range of spacing distributions. Two problem areas were found when the estimated parameters were averaged across samples. First, when the estimated probability of multiples $\left(p_{0}\right)$ was close to zero the estimated variance $\left(\sigma_{0}^{2}\right)$ tended to get yery large. This relationship is to be expected given that a lack of very small spacings can be accounted for by either a very small probability of multiples or a very large variance. Second, when the estimated coefficient of variation $(C V)$ was large the estimated skip probability $(p)$ tended to be small. This relationship may be due to the inability of the model to separate the different gamma distributions when the are poorly defined as in Figure 3.

The results of the analysis for the coefficient of variation, mode, probability of multiples $\left(p_{0}\right)$, and the probability of a skip $(p)$ are summarized in Table I. Planter, speed of operation, and seed type each had significant effects on each of the measure of planter performance. With the exception of the mode, side of the tool bar did not have a significant effect on planter performance.

Least square means for encrusted and pelleted seeds are given in Tables II and III respectively. With very few exceptions it appears that as the speed at which the planter is operated increases the ability of the planter to accurately place seeds decreases. Interactions between planters and speed of operation appears to be in at which speed does planter performance deteriorate. With the exception of the Gaspardo SV255 the planters appear to have less trouble placing pelleted seeds.

\section{Summary}

A model consisting of a mixture of normal and gamma distributions was developed to describe the spatial distribution of sugarbeet plants. The model along with the estimation procedure appears to do a good job in modeling a wide range of spatial distributions that can arise. The estimated parameters from the model provide an objective measure of planter performance. However, it appears that in some situations the estimated density may be biased (e.g. the second peak in Figure 2). The bias may be a result of using samples of 58 spacings to estimate the parameters. In addition, it appears an estimate of the standard errors associated with the estimated parameters are needed.

\section{References}

Abramowitz, M. and Stegun, I. A., eds. (1972), Handbook of mathematical functions with formulas, graphs, and mathematical tables (ninth printing), Dover, Mineola, NY. 
Appendix A. First Partial Derivatives

$$
\begin{aligned}
& \frac{\partial Q(\theta \mid z)}{\partial p_{0}}=\sum_{i=1}^{N}\left\{\frac{\operatorname{Pr}\left(a_{i}>0 \mid z_{i}\right)}{p_{0}}-\frac{\operatorname{Pr}\left(a_{i}=0 \mid z_{i}\right)}{1-p_{0}}\right\} \\
& \frac{\partial Q(\theta \mid z)}{\partial \sigma_{0}^{2}}=\sum_{i=1}^{N} \frac{1}{2 \sigma_{0}^{2}}\left\{\frac{z_{i}^{2}}{\sigma_{0}^{2}}-1\right\} \operatorname{Pr}\left(a_{i}=0 \mid z_{i}\right) \\
& \frac{\partial Q(\theta \mid z)}{\partial p}=\sum_{i=1}^{N}\left\{\frac{\mathrm{E}\left(a_{i} \mid z_{i}, a_{i}>0\right)-1}{p}-\frac{-1}{1-p}\right\} \operatorname{Pr}\left(a_{i}>0 \mid z_{i}\right) \\
& \frac{\partial Q(\theta \mid z)}{\partial \alpha}=\sum_{i=1}^{N}\left\{\frac{1}{2 \alpha}+\left(\ln \left(z_{i} / \beta\right)-\ln (\alpha)\right) \mathrm{E}\left(a_{i} \mid z_{i}, a_{i}>0\right)-\mathrm{E}\left(a_{i} \ln \left(a_{i}\right) \mid z_{i}, a_{i}>0\right)\right\} . \\
& \frac{\partial Q(\theta \mid z)}{\partial \beta}=\sum_{i=1}^{N}\left\{\frac{z_{i}}{\beta^{2}}-\frac{\alpha}{\beta} \mathrm{E}\left(a_{i} \mid z_{i}, a_{i}>0\right)\right\} \operatorname{Pr}\left(a_{i}>0 \mid z_{i}\right)
\end{aligned}
$$

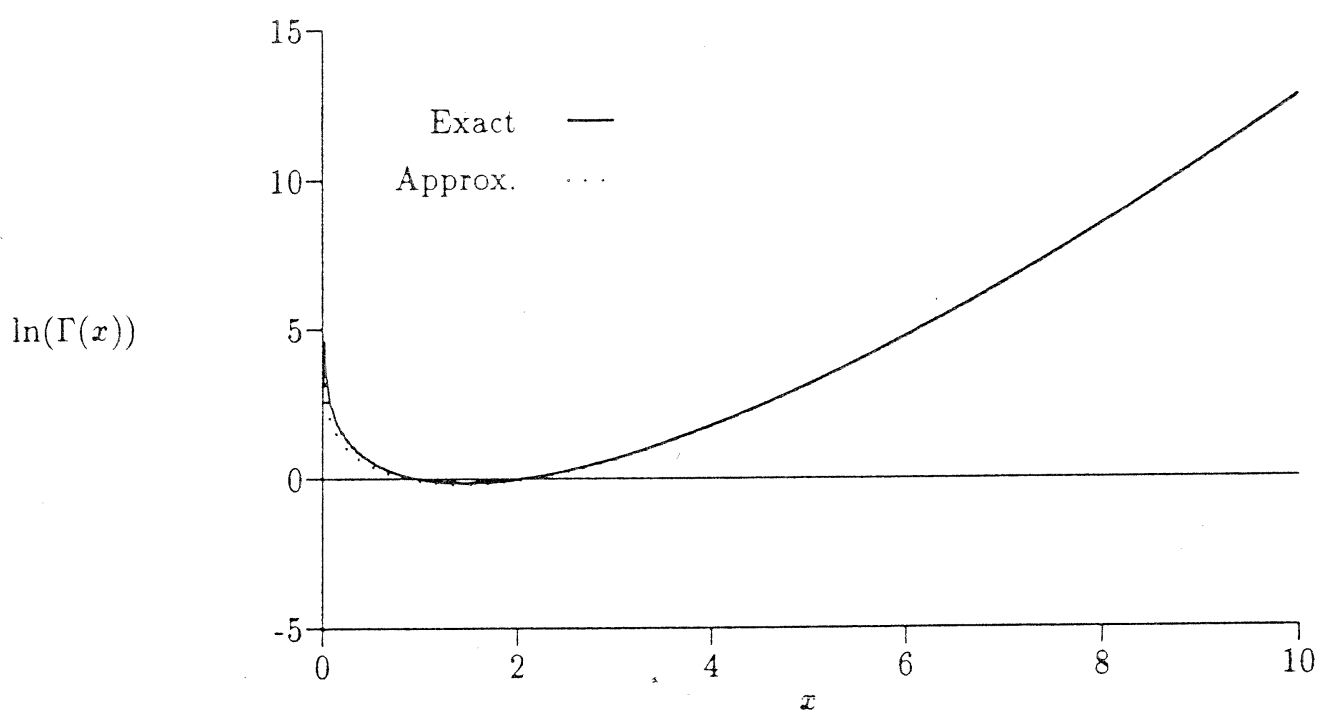

Figure 1: Exact $(\ln (\Gamma(x)))$ versus Approximate $((x-1 / 2) \ln (x)-x+1 / 2 \ln (2 \pi))$. 


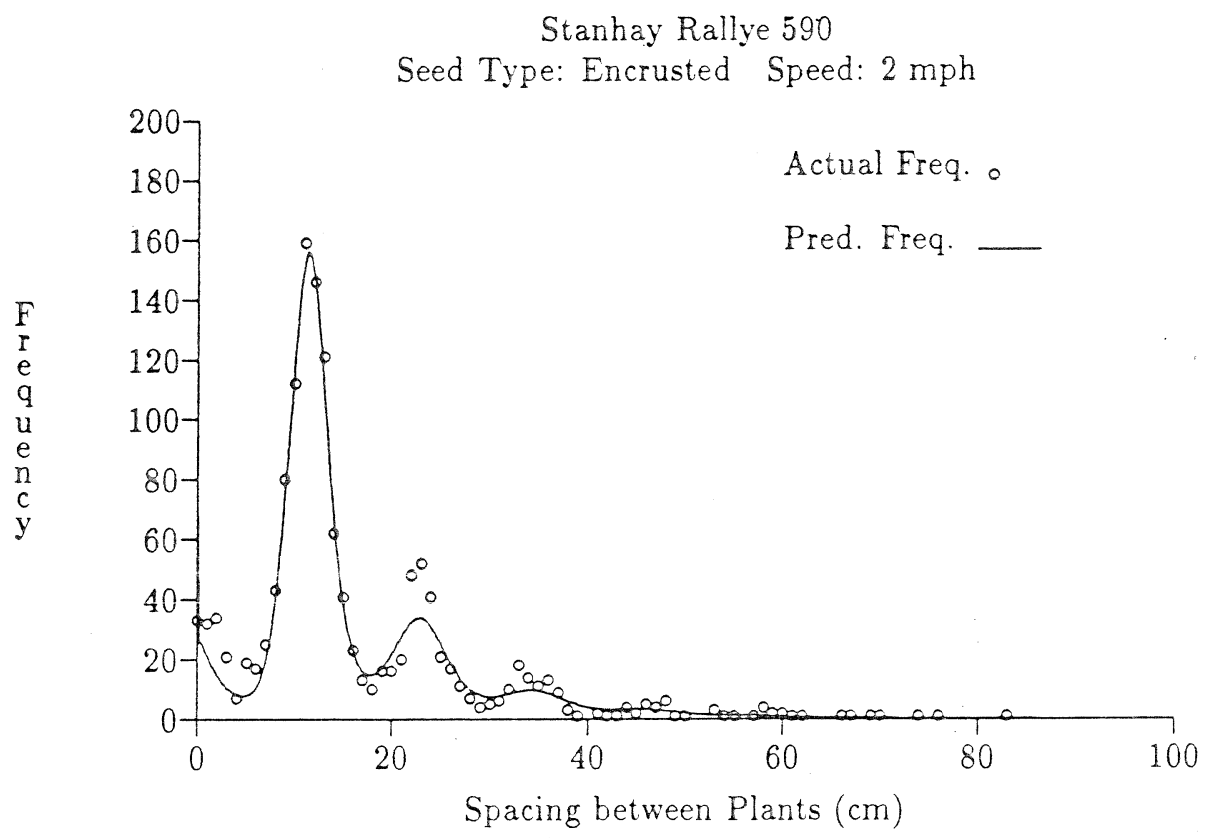

Figure 2: Predicted and actual frequencies for a Stanhay Rallye 590 operated at $2 \mathrm{mph}$ with encrusted seeds

Gaspardo SV255

Seed Type: Pelleted Speed: $5 \mathrm{mph}$

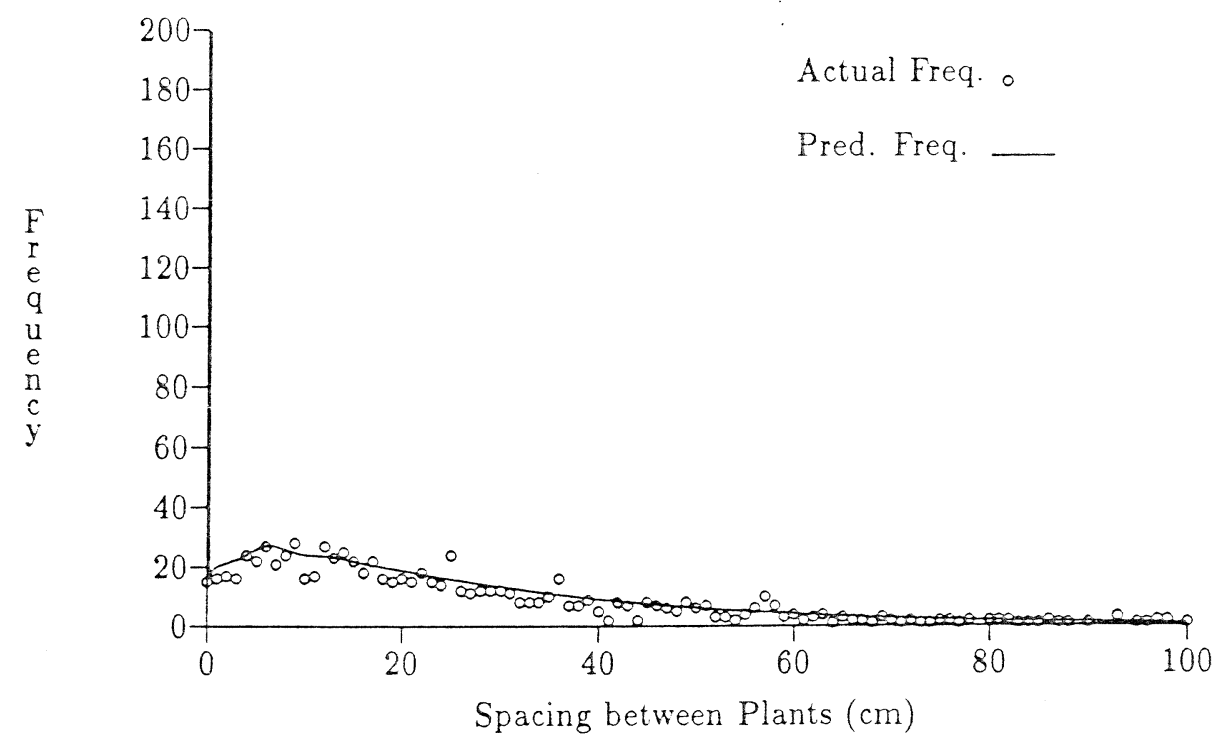

Figure 3: Predicted and actual frequencies for a Gaspardo SV255 operated at $5 \mathrm{mph}$ with pelleted seeds. 
Table I: Analysis of Variance for the 1990 Spacing Study.

\begin{tabular}{|c|c|c|c|c|c|c|}
\hline \multirow[b]{2}{*}{ Source } & & \multirow[b]{2}{*}{ Degrees of Freedom } & \multicolumn{4}{|c|}{$p$-values } \\
\hline & & & $\mathrm{CV}$ & Mode & $P(M u l t)$ & $P($ Skip $)$ \\
\hline Type & (T) & 1 & .0444 & .0110 & .0001 & .0024 \\
\hline $\operatorname{Rep} / \mathrm{T}$ & (R) & 6 & .3766 & .9936 & .2533 & .3381 \\
\hline Planter & (P) & 6 & .0001 & .0001 & .0001 & .0001 \\
\hline $\mathrm{T}^{*} \mathrm{P}$ & & 4 & .0001 & .0003 & .0001 & .0015 \\
\hline Speed & $(\mathrm{Sp})$ & 2 & .0001 & .0031 & .0001 & .0001 \\
\hline $\mathrm{T}^{*} \mathrm{Sp}$ & & 2 & .5466 & .9931 & 0192 & .9124 \\
\hline $\mathrm{P}^{*} \mathrm{Sp}$ & & 12 & .0001 & .0001 & .0016 & .0007 \\
\hline $\mathrm{T}^{*} \mathrm{P}^{*} \mathrm{Sp}$ & & 8 & .7020 & .0009 & .0515 & .1924 \\
\hline Side & (Sd) & 1 & .6738 & .1464 & .5446 & .8884 \\
\hline$T^{*} S d$ & & 1 & .0807 & .0109 & .3669 & .6623 \\
\hline$S p * S d$ & & 2 & .5731 & .9021 & .6101 & .5498 \\
\hline $\mathrm{T}^{*} \mathrm{Sp} \mathrm{p}^{*} \mathrm{Sd}$ & & 2 & .2170 & .7220 & .3311 & .1691 \\
\hline Error & & 180 & & & & \\
\hline
\end{tabular}

Table II: Least Square Means for the 1990 Spacing Study: Seed Type = Encrusted.

\begin{tabular}{llcccc}
\hline \hline \multirow{2}{*}{ Planter } & & \multicolumn{4}{c}{ Least Square Means } \\
\cline { 3 - 6 } & Speed & CV & Mode & $P($ Mult $)$ & $P($ Skip $)$ \\
\hline Stanhay Rallye 590 & 2 & 0.18 & 11.2 & 0.15 & 0.32 \\
& 3.5 & 0.20 & 11.1 & 0.09 & 0.27 \\
Milton & 5 & 0.66 & 10.5 & 0.02 & 0.10 \\
& 2 & 0.37 & 8.7 & 0.17 & 0.14 \\
& 3.5 & 0.34 & 9.2 & 0.15 & 0.17 \\
Deere Maxemerge II & 5 & 0.56 & 8.1 & 0.06 & 0.07 \\
& 2 & 0.38 & 11.6 & 0.11 & 0.21 \\
& 3.5 & 0.47 & 12.0 & 0.06 & 0.10 \\
Deere 71 & 5 & 0.46 & 13.3 & 0.11 & 0.16 \\
& & & & & \\
& 2 & 0.43 & 10.6 & 0.04 & 0.19 \\
Gaspardo SV255 & 3.5 & 0.50 & 11.1 & 0.03 & 0.14 \\
& 5 & 0.68 & 8.4 & 0.03 & 0.05 \\
& 2 & 0.32 & 10.4 & 0.10 & 0.22 \\
White Seedboss & 3.5 & 0.52 & 9.2 & 0.07 & 0.11 \\
& 5 & 0.68 & 8.4 & 0.08 & 0.02 \\
& 2 & 0.59 & 7.8 & 0.12 & 0.08 \\
& 3.5 & 0.67 & 6.0 & 0.07 & 0.09 \\
& 5 & 0.72 & 5.9 & 0.06 & 0.05 \\
\hline \hline
\end{tabular}


Table III: Least Square Means for the 1990 Spacing Study: Seed Type = Pelleted.

\begin{tabular}{|c|c|c|c|c|c|}
\hline \multirow[b]{2}{*}{ Planter } & \multirow[b]{2}{*}{ Speed } & \multicolumn{4}{|c|}{ Least Square Ḿeans } \\
\hline & & $\mathrm{CV}$ & Mode & $P(M u l t)$ & $P($ Skip $)$ \\
\hline \multirow{3}{*}{ Stanhay Rallye 590} & 2 & 0.12 & 11.3 & 0.05 & 0.34 \\
\hline & 3.5 & 0.20 & 11.4 & 0.03 & 0.29 \\
\hline & 5 & 0.61 & 13.7 & 0.04 & 0.17 \\
\hline \multirow[t]{3}{*}{ Milton } & 2 & 0.26 & 10.2 & 0.04 & 0.24 \\
\hline & 3.5 & 0.30 & 10.3 & 0.07 & 0.29 \\
\hline & 5 & 0.47 & 10.6 & 0.03 & 0.28 \\
\hline \multirow[t]{3}{*}{ Deere Maxemerge II } & 2 & 0.28 & 12.4 & 0.05 & 0.28 \\
\hline & 3.5 & 0.25 & 12.4 & 0.04 & 0.33 \\
\hline & 5 & 0.52 & 12.0 & 0.03 & 0.14 \\
\hline \multirow[t]{3}{*}{ Stanhay S870 Belt } & 2 & 0.22 & 8.9 & 0.06 & 0.38 \\
\hline & 3.5 & 0.48 & 8.7 & 0.02 & 0.24 \\
\hline & 5 & 0.57 & 8.2 & 0.02 & 0.24 \\
\hline \multirow[t]{3}{*}{ Deere 71} & 2 & 0.29 & 10.1 & 0.03 & 0.25 \\
\hline & 3.5 & 0.39 & 10.5 & 0.03 & 0.13 \\
\hline & 5 & 0.56 & 9.4 & 0.04 & 0.06 \\
\hline \multirow[t]{3}{*}{ Gaspardo SV 255} & 2 & 0.45 & 10.3 & 0.04 & 0.21 \\
\hline & 3.5 & 0.68 & 9.8 & 0.06 & 0.03 \\
\hline & 5 & 0.83 & 5.1 & 0.03 & 0.07 \\
\hline
\end{tabular}

